\title{
Uso da Oscilação Oral de Alta Freqüência em Pacientes Ventilados Mecanicamente, um Estudo Prospectivo e Revisão de Literatura
}

\section{The Use of the Oral High Frequency Oscillation in Mechanically Ventilated Patients, a Study Prospective and Literature Revision}

\author{
Ana Cristina Tavares Silveira ${ }^{1}$ \\ Cleize Silveira Cunha ${ }^{2}$ \\ Danielle Batista Pacheco ${ }^{3}$ \\ Ney Marcelo de Azevedo Silva ${ }^{4}$
}

\section{Resumo}

A Oscilação Oral de Alta Freqüência (OOAF), ou Oral High Frequency Oscillation (OHFO), vem sendo estudada como um novo recurso fisioterapêutico, desde a década de 80 , como sendo uma técnica de desobstrução brônquica. Para GAVA e ORTENZI (1998), a OOAF pode ser utilizada como coadjuvante à fisioterapia respiratória no tratamento de doenças pulmonares que se caracterizam pelo aumento de secreção brônquica. É atribuído a OOAF o descolamento da secreção brônquica, o aumento da função pulmonar e a melhora da oxigenação. Diversos autores descrevem sobre os aparelhos que estão disponíveis no mercado. Contudo, apresentam uma forma básica que lembra um pequeno cachimbo, em que, no seu interio,r existe um canal, onde está acentuada uma pequena esfera de aço. Na realidade, é um fiel resistor de limiar pressórico gravitacional, o qual permite a frenagem do fluxo expiratório, por produzir curtas e sucessivas interrupções à passagem do fluxo, que permitem uma repercussão oscilatória produzida por resistor do aparelho, que é transmitida à árvore brônquica. Atualmente, existem três modelos distintos no mercado: Flutter, Shaker e Acapella. (VOLSKO et al 2003). CARVALHO (2001) ressalta que a pressão positiva oscilatória,atua promovendo a dilatação dos brônquios até as estruturas periféricas, facilitando deslocamento do muco e inibindo o colapso precoce brônquico, tendo como conseqüência, uma diminuição da resistência respiratória e o aumento do volume de reserva expiratória. Estudamos prospectivamente, 14 pacientes, de ambos os sexos, com idade variando de 22 a 71 anos, portadores de vários tipos de doenças, tendo em comum o aumento da secreção brônquica. O presente estudo foi realizado na Unidade de Terapia Intensiva do Hospital VITA Volta Redonda.

Palavras-chave: Fisioterapia, Pacientes, Melhora.

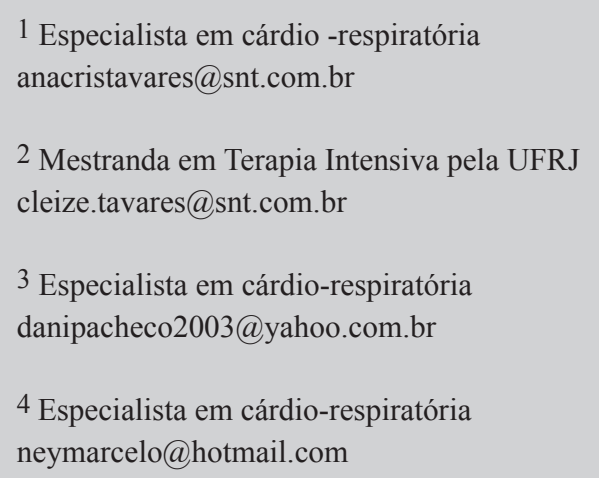




\section{Abstract}

The Oral High Frequency Oscillation (OHFO) are being studied as a new phisiotherapeutic resource, since the 80's, as being a technique of bronchi obstruction. For GAVA and ORTENZI (1998), the OHFO can be used as a coadjuvant to the respiratory physiotherapy in the treatment of pulmonary illnesses that is characterized for the bronchi secretion increase. It is attributed to the OHFO the dislocation of the bronchi secretion, the increase of the pulmonary function and the improvement of the oxygenation. Assorted authors describe on the devices that are available in the market. However, they present a basic form that remembers small tobacco-pipe, where, in its inner exists a channel, where a small steel sphere is accented. In fact, it is a faithful electrical resistance of gravitational threshold, which allows the lockwire of the expiratory flow, for reducing shortnesss and successive interruptions to the fleeting of the flow, that they allow an oscillatory repercussion produced by electrical resistance of the device, that is transmitted the bronchi tree. Currently, there are three distinct models in the market: Flutter, Shaker and Acapella. (VOLSKO et al 2003). CARVALHO (2001) stands out that the oscillatory positive pressure, acts promoting the dilatation of the bronchis until the peripheral structures, facilitating displacement of mucous and inhibiting the bronchi precocious collapse, having as a consequence, a reduction of the respiratory resistance and the increase of the volume of expiratory reserve. We study prospectively, 14 patients, from both sexes, with age varying of 22 until 71 years, carriers of some types of illnesses, having in common the increase of the bronchi secretion. The present study was carried through in the Intensive Therapy Unit of the VITA Volta Redonda Hospital.

Keywords: Physiotherapy, Patients, Improvement. 


\section{OOAF como técnica fisioterapêutica}

AMARAL (2003) define a OOAF como uma aplicação terapêutica periódica intermitente de resistor limiar pressórico do tipo gravitacional à fase expiratória do paciente. Produz uma frenagem do fluxo expiratório pela produção de curtas e sucessivas interrupções na passagem deste. SLUTZKY (1997) acrescenta que a aplicação do resistor e a frenagem do fluxo permitem uma repercussão oscilatória de alta freqüência a qual é transmitida à região traqueal e a árvore brônquica, fazendo que ocorra o descolamento e o deslocamento das secreções traqueobrônquicas. SCALAN et al (2000) definem a OOAF como a oscilação que se refere ao movimento vibratório rápido de pequenos volumes de ar para frente e para trás no trato respiratório que, em altas freqüências (12 a $25 \mathrm{~Hz}$ ), as oscilações atuam como um "mucolítico físico", acentuando a tosse com eliminação de secreções. CHATAM et al (1993) descrevem que a resistência produzida pela bola de aço, durante a expiração, produz um nível de pressão positiva nas vias aéreas (EPAP) ou uma pressão positiva expiratória (PEP) e podem atingir até 20 $\mathrm{cmH} 2 \mathrm{O}$, e uma oscilação intratraqueal em torno de 6 a $20 \mathrm{~Hz}$. No período da fase expiratória, a posição da bola de aço depende do equilíbrio do ar expirado, da força da ação da gravidade sobre a bola e do ângulo de contato com o canal cônico do aparelho, o ar pode, escapar após o aumento da pressão dentro do canal cônico pelas aberturas de forma de pequenos ou grandes furos na extremidade do aparelho. PRESTO e PRESTO (2003) relatam que, à medida que a bola metálica cai, ela retorna à posição inicial de repouso, bloqueando o canal cônico e criando outra configuração de uma outra determinada pressão. Há diminuição da aderência das secreções à parede brônquica, com conseqüente deslocamento e mobilização do muco, o que reduz zonas de obstruções brônquicas (GAVA e ORTENZI, 1998).

A posição adotada para realização da OOAF é a sentada, com inclinação anterior do corpo em 30 graus, e, em casos de pacientes no leito, a posição inicial é a Fowler, ressaltando que a inclinação anterior deverá ser analisada de acordo com o estado clínico e condições gerais do paciente. O tempo de aplicação da terapia vem sendo limitado entre 10 e 15 minutos diários. A nosso ver, a técnica deverá ser indicada com um período inicial de 3 minutos, evoluindo gradativa- mente, atingindo um limite máximo de 15 a 35 minutos, variando de acordo com cada doença. A terapia inalatória deve ser, sempre que possível, realizada em conjunção com a OOAF, com os objetivos de fluidificação prévia das secreções e broncodilatação das vias aéreas; A combinação da OOAF com outras técnicas fisioterápicas deve ser, sempre que possível, preconizada pelo terapeuta responsável, pois isso poderá potencializar os efeitos benéficos da terapia. FERRO (2003) comparou a terapia com a OOAF com a terapia convencional e concluiu que o tratamento com a OOAF foi mais eficiente e também o mais preferido pelos pacientes.

\section{Flutter - Modelo VRP1}

Flutter é um aparelho de fabricação suíça, já utilizado em vários centros europeus e no Brasil. Destina-se a melhorar a ventilação pulmonar, diminuir a dispnéia e, especialmente, facilitar e promover a expectoração, quando é gerada uma pressão positiva oscilatória controlada com interrupções do débito expiratório a freqüência regulável. Segundo SCALAN et al (2000), a válvula de flutter combina as técnicas do EPAP com as oscilações de alta freqüência na abertura das vias aéreas. A válvula é composta por um dispositivo em forma de cachimbo com uma bola de aço pesada localizada numa "cabeça" angulada. Quando o paciente expira ativamente no interior do cachimbo, faz com que a válvula tremule para frente e para trás em aproximadamente $15 \mathrm{~Hz}$. Quando a válvula é utilizada adequadamente, as oscilações que ela cria são transmitidas para baixo, para o interior das vias aéreas. Para PRYOR et al (2002), o flutter é um aparelho portátil pequeno, com uma abertura única na peça bucal e uma série de pequenos furos na saída na extremidade da estrutura arredondada, contendo uma bola de aço inoxidável incluída num pequeno cone. Durante a expiração, o movimento da bola, ao longo da superfície do cone, cria uma pressão expiratória positiva (PEP) e uma oscilação vibratória do ar dentro das vias aéreas. O aparelho é mantido na porção horizontal e inclinado levemente para baixo até que se sinta um máximo efeito oscilatório. TOMPSON et al (2002) relatam que o aparelho de flutter impede o fechamento prematuro das vias aéreas descolando e deslocando secreções. 


\section{Acapella}

Acapella é um novo aparelho desenvolvido para higiene brônquica, que funciona de maneira similar ao flutter. AZEREDO et al (2002) descrevem que o mecanismo de ação do Acapella é a vibração com pressão positiva expiratória, ou seja, pressão oscilatória positiva intrabrônquica durante a expiração, com a vantagem de poder executar a terapia em qualquer posição e com freqüência ajustável, o que o difere do flutter é que deverá manter uma relação de perpendicularidade com o solo. Segundo VOLSK et al (2003), o Acapella combina princípios de oscilação de alta freqüência e PEP, empregado por uma alavanca e um imã contrapesador, o ar passa através de um cone produzindo oscilações do fluxo. O Acapella está disponível no mercado em dois modelos: Green: para pacientes que alcançam um fluxo expiratório de 15 1/min ou mais e conseguem mantê-lo por 3 segundos; Blue: para pacientes que não conseguem manter um fluxo expiratório de $15 \mathrm{l} / \mathrm{min}$. A freqüência obtida varia de 0 a $30 \mathrm{~Hz}$ na maioria dos pacientes.

\section{Shaker}

A empresa NCS de São Paulo, com base nos resultados obtidos com o emprego do Flutter, lançou, em setembro de 2002, um protótipo nacional denominado Shaker. É um cachimbo que, no seu interior, possui uma bilha de metal em cima de um cone (PRESTO e PRESTO, 2003). COSTA et al (2002), no Laboratório de Espirometria e Fisioterapia Respiratória (UFSCar - São Carlos, SP), realizaram um experimento com o Shaker a partir do fato que a associação das Pressões Expiratórias Positivas com vibrações favorecem o aumento da permeabilidade das vias aéreas, diminuindo a dispnéia e facilitando a expectoração. $\mathrm{O}$ estudo foi realizado com objetivo de comparar os efeitos do equipamento Flutter com Shaker, por intermédio das medidas de saturação de oxigênio $(\mathrm{SpO} 2)$, de Peak Flow (PF), de freqüência cardíaca (FC), de freqüência respiratória (FR), de pressão arterial (PA) e de escala de Borg (EB), em pacientes com doenças pulmonares. Compuseram a amostra 14 pacientes, com idade de $67 \pm 5$ anos, divididos aleatoriamente em dois grupos: G1 (Flutter) e G2 (Shaker). Todos foram submetidos ao procedimento experimental que constaram de 1 a 5 sessões consecutivas de nebulização, seguidas de 5 séries de 10 repetições de exercícios expiratórios no equipamento. Foram coletadas medidas de $\mathrm{SpO} 2 \mathrm{e}$ FC na $5^{\text {a }}$ repetição de cada série e nas pausas, quando também foi medido o Peak Flow. Antes e após esse procedimento, mediram-se a $\mathrm{SpO} 2$, a FC, a fr, a $\mathrm{PA}$ e a EB.Constatou-se que os equipamentos Flutter e Shaker não apresentaram diferenças significativas quanto aos efeitos estudados.

\section{Indicações e Contra-Indicações para Utilização da Oscilação Oral de Alta Freqüência (OOAF)}

\subsection{Indicação para o uso da OOAF}

Poderemos indicar a terapia nos casos de secreção produtiva persistente e tosse não produtiva, bronquite aguda e crônica, asma associada à obstrução brônquica, bronquiectasia, instabilidade traqueobrônquica; enfisema pulmonar, fibrose cística, pré e pósoperatórios em geral. SCALAM et al (2000) acrescentam as síndromes ciliares discinésicas. MILLER (1993), completa com asma fora das crises.GAVA e ORTENZI (1998) analisaram trabalhos clínicos e concluíram que a utilização da OOAF não substitui a fisioterapia convencional, mas sua utilização está indicada em alguns casos em que as manobras de higiene brônquica causem dor e, muitas vezes, são contra-indicadas.

\subsection{Contra-Indicações para o uso da OOAF}

Em qualquer paciente adulto ou criança que não consiga gerar fluxo expiratório suficiente para produzir níveis ideais de oscilação, broncoespasmo, doença cardíaca descompensada, fraturas faciais instáveis e erosões por queimaduras, fraturas escalonadas de arcos costais, grandes queimaduras, pacientes hemodinamicamentes instáveis, alterações renais graves, traumatismo torácico grave, tuberculose pulmonar em atividade, pneumotórax sem drenagem prévia, derrame pleural sem drenagem prévia. SCALAN et al (2000) afirmam que não foram relatadas contra-indicações absolutas, por isso os seguintes itens devem ser cuidadosamente avaliados antes do início da terapia: Pacientes incapazes de tolerar o aumento do trabalho respiratório (asma aguda, DPOC), pressão intracra- 
niana $(\mathrm{PIC})>20 \mathrm{mmHg}$, instabilidade hemodinâmica, sinusite aguda, hemoptise ativa, pneumotórax não tratado, ruptura de membrana timpânica ou doença de ouvido médio, cirurgia ou traumatismo facial, oral ou craniana recente, epistaxe, cirurgia esofágica.

\section{OOAF no Ventilador Mecânico - Estudo Pros- pectivo}

Foram avaliados prospectivamente, na Unidade de Terapia Intensiva do Hospital VITA Volta Redonda, 14 pacientes de ambos os gêneros, faixa etária variando de 22 a 71 anos, com doenças diversificadas, tendo em comum o aumento da secreção brônquica. Todos os pacientes encontravam-se entubados ou traqueostomizados, ventilados mecanicamente, através do ventilador mecânico Inter-5 da Intermed. Adaptamos o SHAKER na válvula expiratória do ventilador mecânico, por um período de, no máximo, 20 minutos, por 4 vezes ao dia. A opção pelo SHAKER e não por outros OOAF, se deve ao fato desse aparelho possuir uma conexão a qual denominamos, universal, pois facilita a adaptação na válvula expiratória dos ventiladores mecânicos. Alguns pacientes eram retirados antes do período de tempo previsto, devido à presença do reflexo de tosse e deslocamento da secreção, esses pacientes necessitavam de toillete brônquica imediata. Observamos que os pacientes permanecem mais bem adaptados à terapia quando sedados e/ou curarizados, portanto, no modo ventilatório controlado e com a sensibilidade aumentada, para não haver disparo do ventilador mecânico pelo paciente ou auto-ciclagem do ventilador mecânico e, em nível ZEEP, para não haver aumento da resistência expiratória. Ressaltamos a não utilização do procedimento em pacientes PEEP dependentes. Monitoramos, durante e após a técnica, saturação de $\mathrm{O} 2$, freqüência cardíaca, freqüência respiratória e ausculta pulmonar. Os resultados encontrados foram bastante satisfatórios, ocorrendo o deslocamento da secreção e como conseqüência melhora dos parâmetros previamente avaliados.

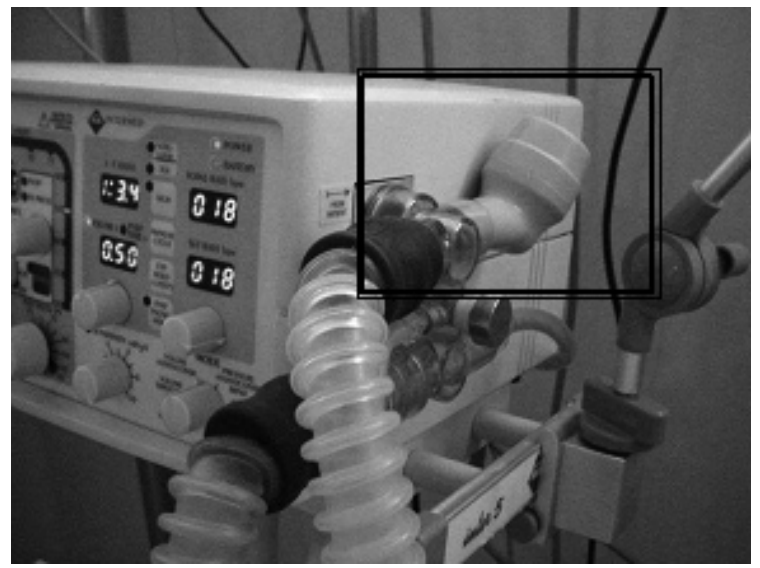

Figura 1 - SHAKER acoplado ao ventilador mecânico Inter-5, através da válvula expiratória

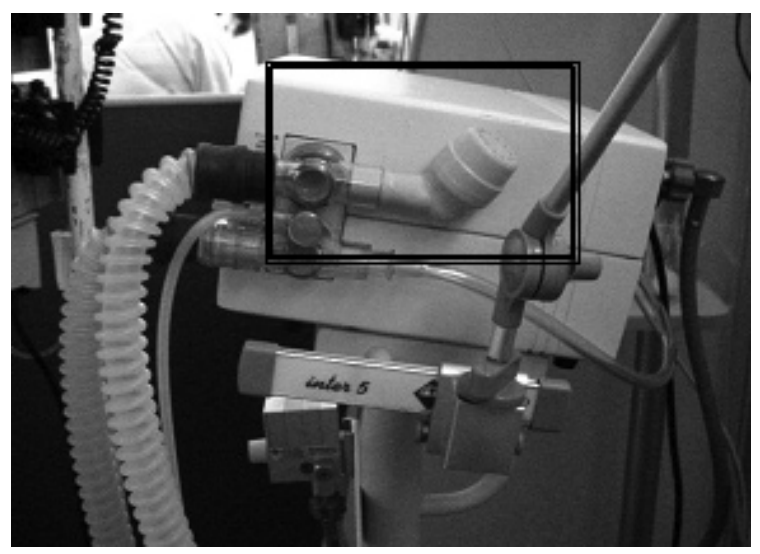

Figura 2 - Vista lateral do SHAKER adaptado à válvula expiratória do Inter-5

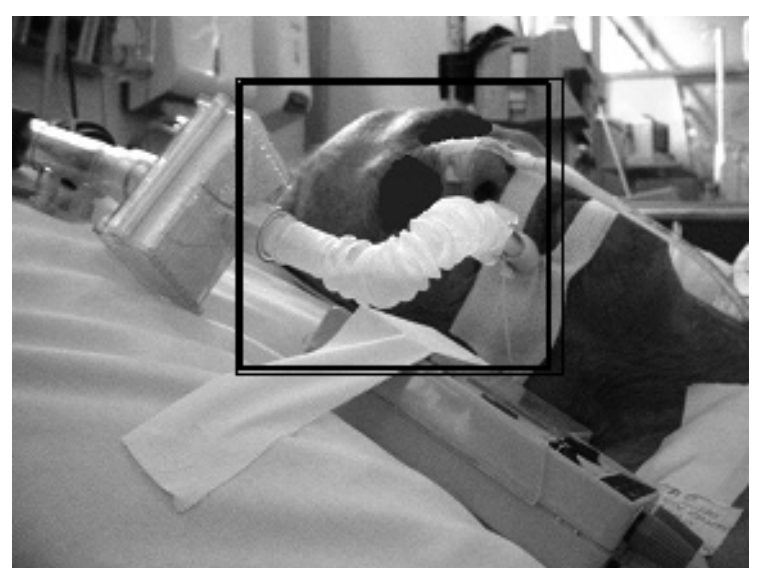

Figura 3 - Deslocamento da secreção, durante a realização da técnica 


\section{Conclusão}

Podemos verificar que a Oscilação Oral de Alta Freqüência (OOAF) é utilizada na fisioterapia no tratamento de doenças pulmonares que se caracterizam por aumento de secreção brônquica, a aplicação do resistor e a frenagem do fluxo permitem uma repercussão de oscilatória de alta freqüência a qual é transmitida à região traqueal e a árvore brônquica, fazendo que ocorra o descolamento e o deslocamento das secreções traqueobrônquicas.

A pesquisa que foi realizada no Hospital
VITA Volta Redonda, com base na fundamentação teórica e na possibilidade da acoplar o aparelho de OOAF (Shaker) ao ventilador mecânico Inter-5, nos demonstra que a terapia é eficaz e os resultados são satisfatórios, sendo necessária a toillete brônquica desses pacientes de acordo com o deslocamento da secreção que ocorre para a via aérea mais proximal e facilitando a desobstrução brônquica.

Consideramos que essa terapia é mais um coadjuvante ao tratamento fisioterapêutico na Unidade de Terapia Intensiva, para pacientes que apresentam acúmulo de secreção em vias aéreas.

\section{Referências}

AMARAL, Marcus Vinícius. Utilização da Pressão Expiratória Positiva Oscilante através de um Novo Equipamento Denominado “Shaker”. Disponível em: http//:www.fisiocor.com.br. Acesso em 01/10/2003.

AZEREDO, Carlos Alberto Caetano. Fisioterapia Respiratória Moderna. 4. ed. Rio de Janeiro: Manole, 2002.

CARVALHO, Mercedes. Fundamentos Respiratórios: Fundamentos e Contribuições. 5. ed. Rio de Janeiro: Revinter, 2001.

CHATHAM, K.; MARSHALL, C.; CAMPBELL, A.; PRESCOTT, R.J. The Flutter VRP1 Device for Pos Thoracotomy Patient. Physiotherapy, v.79, p.95-99, 1993.

COSTA, D.; MARTINS, A.L.P.; JAMAMI, M. Comparação entre os Equipamentos Flutter e Shaker em Pacientes Portadores de Doenças Pulmonares. Revista Brasileira Fisioterapia. Agosto/2002 suplemento. p.71.

FERRO, Aline Torres. Estudo Comparativo sobre Técnicas Fisioterápicas para Desobstrução Brônquica. Disponível em: http://www.interfisio.com.br. Acesso em: 09/03/2004.

GAVA, Marcus Vinícius; ORTENZI, Luciana. Estudo analítico dos efeitos fisiológicos e da utilização do aparelho Flutter URP1. v. XI, nº 1, abril/setembro 1998.

MILLER, J.J. Indicações do Flutter. I Congresso Brasileiro de Fisioterapia em Terapia Intensiva. Porto AlegreRS, Outubro, 1993.

PRESTO, Bruno; PRESTO, Luciana Damázio de Noronha. Fisioterapia Respiratória: Uma Nova Visão. 1.ed. São Paulo: Bruno Presto, 2003.

PRYOR, Jennifer A.; WEBBER, Bárbara A. Fisioterapia para Problemas Respiratórios e Cardíacos. 2. ed. Rio de Janeiro: Guanabara Koogan, 2002.

SLUTZKY, Luis Carlos. Fisioterapia Respiratória - Nas Enfermidades Neuromusculares. 1.ed. Rio de Janeiro: Revinter, 1997. 
SCALAN, Craig L.; WILKINS, Robert L.; STOLLER, James K. Fundamentos da Terapia Respiratória de EGAN. Seção 6. p.817-839, 7.ed. 2000.

TOMPSON, C.S.; HSRRISON, S.; ASHLEY, J.; DAY, K.; SMITH, D.L.. Randomised crossover study of the Flutter device and active sycle of breathing techinique in non - cystic fibrosis. Bronquiestasis. torase, 200; 57:446-448.

VOLSKO, A. Teresa; DIFRORE, Juliann M.; CHATBURN, Robert L. Performance comparison of two Oscillating Positive Expiratory. Pressure Device: Acapella Versus Flutter. Respiratory Care, Vol. 48, n 2, Fevereiro, 2003.

\section{Informações bibliográficas:}

Conforme a NBR 6023:2002 da Associação Brasileira de Normas Técnicas (ABNT), este texto científico publicado em periódico eletrônico deve ser citado da seguinte forma:

SILVEIRA, A. C. T.; CUNHA, C. S.; PACHECO, D. B.; SILVA, N. M. A. Uso da Oscilação Oral de Alta Freqüência em Pacientes Ventilados Mecanicamente, um Estudo Prospectivo e Revisão de Literatura. Cadernos UniFOA, Volta Redonda, ano II, n. 4, agosto. 2007. Disponível em: $<$ http://www.unifoa.edu.br/pesquisa/caderno/edicao/04/104.pdf> 